\title{
Evaluation of Health Promotion Standards in the Iraqi Teaching Hospitals: A National Survey
}

\author{
Mohammed Baqer Habeeb Abd Ali, M.Sc ${ }^{1}$, Naji Yasser Saadoon $\mathrm{PhD}^{2}$, \\ Hewa Sattar Salih, $\mathrm{PhD}^{3}$ \\ I(Instructor, Community Health Nursing Department, College of Nursing/ University of Baghdad, Iraq) \\ ${ }_{2}^{2}$ (Instructor, Community Health Nursing Department, College of Nursing/ University of Babylon, Iraq \\ ${ }_{3}^{3}$ (Instructor, Clinical Nursing Science Department, College of Nursing/University of Kirkuk, Iraq
}

\begin{abstract}
The objective of this survey is to explore the reality of the the health promotion standards in the governmental teaching hospitals in Iraq. The researchers used the Health Promotion Self-Assessment Questionnaire for Companies to measure the health promotion standards in these hospitals. The study sample included nurses and physicians from 42 hospitals across Iraq. The study findings demonstrated that the vast majority of governmental teaching hospitals in Iraq lack the health promotion standards and considered as nonhealth promoting. The standards of health promotion objectives and health promotion planning had the highest scores. Only two hospitals out of 62 hospitals considered to be health promoting ones.
\end{abstract}

Keywords: Health Promotion Standards, Teaching Hospitals

\section{Introduction}

The World Health Organization's (WHO) Ottawa Charter for Health Promotion; as a primary response to growing expectations for a new public health movement around the world, led to the development of a series of 'settings-based' (1). The Health Promoting Hospitals (HPH) project and network began in 1988 and 1993, respectively, to promote the total quality management of hospitals. The project also aims at addressing the health of staff and the link of the hospital to its community ${ }^{(1)}$. Health promotion is defined in the Ottawa Charter as "the process of enabling people to increase control over, and improve, their health" (1).

Health promotion is understood to embrace health education, disease prevention and rehabilitation services, but stresses that information, education and advice only lead to sustained behavioral change if supported by prevailing norms, rules and cultures. Health promotion interventions in organizations therefore have to address these underlying causes ${ }^{(2)}$.

Hospitals are an important component of the health care system and are central to the process of reform, and yet, as institutions, they have received remarkably little attention from policy-makers and researchers ${ }^{(3)}$. They are important within the health care system for several reasons. First, they account for a substantial proportion of the health care budget. Second, their position at the apex of the health care system means that the policies they adopt, which determine access to specialist services, have a major impact on overall health care. Third, the specialists who work in hospitals provide professional leadership. Finally, technological and pharmaceutical developments, as well as more attention to evidence-based health care, mean that the services that hospitals provide can potentially contribute significantly to population health ${ }^{(4)}$. If hospitals are ineffectively organized, however, their potentially positive impact on health will be reduced or even be negative (3).

There is large scope and public health motivation for offering health promotion strategies in health care settings ${ }^{(2)}$. Hospitals consume between $40 \%$ and $70 \%$ of the national health care expenditure and typically employ about $1 \%$ to $3 \%$ of the working population ${ }^{(3)}$. These working places are characterized by certain physical, chemical, biological and psychosocial risk factors. Paradoxically, in hospitals—organizations that aim to restore health - the acknowledgement of factors that endanger the health of their staff is poorly developed. Hospitals can also have a lasting impact on influencing the behavior of patients and relatives, who are more responsive to health advice in situations of experienced ill-health ${ }^{(2)}$.

Hospitals produce high amounts of waste and hazardous substances ${ }^{(2)}$. Introducing health promotion strategies to hospitals can help to reduce the pollution of the environment and cooperation with other institutions and professionals can help to achieve the highest possible coordination of care. Furthermore, as research and teaching institutions, hospitals produce accumulate and disseminate a lot of knowledge and can have an impact on the local health structures and influence professional practice elsewhere ${ }^{(2)}$.

A health promoting hospital does not only provide high quality comprehensive medical and nursing services, but also develops a corporate identity that embraces the aims of health promotion, develops a health promoting organizational structure and culture, including active, participatory roles for patients and all members 
of staff, develops itself into a health promoting physical environment, and actively cooperates with its community ${ }^{(5)}$.

Fundamental principles dictate that HPHs should improve well-being and empower health professionals, patients, their relatives and the community with holistic healthcare that acknowledges the diverse needs and values of different population groups, and both protects and improves the hospital environment and greater community. Quality care and improvement of hospital 'settings' and culture should go beyond curative services to health promotion through participatory, 'health-gain-oriented procedures' including changes to: communication, training, education, organizational management, policy development; and the fostering of close links between hospitals and the community ${ }^{(6)}$.

The history of the health system in Iraq began in the early twenties of the 20th century. During the 1970s and early 1980s, Iraq experienced improvements in several critical health outcomes ${ }^{(7)}$. The health care system - a hospital-oriented, capital-intensive model that requires largescale imports of medicines, medical equipment and even health workers - is inefficient and access is inequitable. Although the system ran fairly effectively, little health service data was collected. This led to a lack of cost-effective public health interventions, and services only partially matched population health needs. To this day, the levels and distribution, of available human resources for health is inadequate ${ }^{(7)}$.

The Iraqi healthcare system is mainly central, with assured allocation of government subsidy dedicated towards the sector annually. As stated by the World Health Organization, there are 1,146 primary health centers run by mid-level workers; and 1,185 health centers, run by medical doctors. There are 229 hospitals, involving 61 teaching hospitals. Government disbursements on healthcare has raised in the last decade, as stated by the World Bank: In 2003 disbursements was at $2.7 \%$ of GDP, and in 2010 it had hurdled to $8.4 \%{ }^{(8)}$.

The predominant approach to quality management in hospitals is through setting standards for the services. Health promotion is a core quality issue for improving health and sustaining quality of life. The standards for health promotion in hospitals are necessary to ensure the quality of services provided in the area of health promotion activities. This study is the first one that is conducted in Iraq to investigate this area.

\section{Methods}

Aim: This study aims to investigate the level of the health promotion achievement in the Iraqi teaching hospitals.

Setting: The study was conducted in teaching hospitals in Iraqi governorates.

Sample: The study sample included a purposive "non-probability" sample of 42 nurses and physicians who have been working in Iraqi teaching hospitals. These hospitals were selected from 15 governorates in Iraq (out of 18 governorates; three are unstable in security situation) as follows: Baghdad ( 9 hospitals), Missan (3 hospitals), Al-Muthanna (2 hospitals), Al-Najaf (2 hospitals), Babylon (2 hospitals), Basrah (2 hospitals), Diyala (2 hospitals), Dohuk (3 hospitals), Erbil (3 hospitals), Karbala (2 hospitals), Kirkuk (3 hospitals), Sulaimaniyah (3 hospitals), Thi Qar (2 hospitals), Wasit (2 hospitals) and Al-Diwaniyah (2 hospitals).

Study Instrument: The researchers used the Health Promotion Self-Assessment Questionnaire for Companies established by National Centre for Workplace Health Promotion Nofer Institute of Occupational Medicine Lodz, Poland. This tool has been developed in order to determine whether the health promotion standards have been already met in a given institution. The scoring of this tool includes ( 2 points) for the described standard occurs in the institution, ( 1 point) when there are already plans and some effort made to achieve the described 'ideal' situation, and ( 0 points) for no plans have been yet prepared and no other activities have been completed concerning the respective standard. The total score can be calculated by summing all the points. The more points a given institution scores; the better the quality of its workplace health promotion program. For a workplace to be considered a health-promoting 'a model of good practice' it should get 24 points and more, but an institution has to have at least 4 points in each of the 6 standards. This means that an institution with a good practice has reached in all the categories at least one of the described standards and is preparing to fulfil the two remaining ones or has implemented the proper approach in two areas out of three. A back-translation for the study instrument has been made by two bilinguists; who are well knowledgeable about both original and target languages, truly bilingual and familiar with the area under study in the source materials; from the target language (Arabic) back to the source language (English) in order to verify translation of the research instrument (9). The equivalence between source and target versions has been evaluated. The content of the items has been established. Back-translation procedures should be used to the test instruments in addition to the items themselves ${ }^{(10-11)}$. Testing of both source and target language versions revealed that items yielding no discrepant responses in the two versions.

Data Analysis: Data were analyzed by using the Statistical Package for Social Science (SPSS) for Windows, version 16. Descriptive statistics (frequency, percent, Mean, and standard deviation) were used in this study. 


\section{Results}

Table 1. Participants' Socio-demographic and Employment Characteristics $(\mathrm{N}=42)$

\begin{tabular}{|l|l|l|l|}
\hline List & Variable & Frequency & Percent \\
\hline 1. & Age: Mean (SD) $=37.5 \pm 8.7$ & 17 & \\
& $25-32$ & 8 & 40.5 \\
& $33-39$ & 9 & 19.0 \\
& $40-46$ & 8 & 21.4 \\
& $47-54$ & & 19.0 \\
\hline 2. & Gender & 20 & \\
& Female & 22 & 47.6 \\
& Male & & 52.4 \\
\hline 3. & Job title & 18 & \\
& Associate Nurse & 20 & 42.9 \\
& BSN Nurse & 4 & 47.5 \\
& Physician & & 9.5 \\
\hline 4. & Level of education & 18 & \\
& Diploma & 24 & 42.9 \\
& Bachelor & & 57.1 \\
\hline 5. & Years of employment: Mean (SD) $=13.7 \pm 8.5$ & 10 & \\
& $\leq 6$ & 8 & 23.8 \\
& $7-10$ & 7 & 19.0 \\
& $11-15$ & 17 & 16.7 \\
& $\geq 16$ & & 40.5 \\
\hline 6. & Years of working in current unit: Mean (SD) $=5.1 \pm 3.4$ & 25 & \\
& $\leq 5$ & 15 & 39.5 \\
& $6-10$ & 2 & 35.7 \\
\hline & $\geq 11$ & & \\
\hline
\end{tabular}

The Mean age is $37.5 \pm 8.7$; about two fifth are within the $25-32$ old-age group $(n=17 ; 40.5 \%)$, followed by more than a fifth who are in the 40-46 years-old age group ( $n=9 ; 21.4 \%)$, less than a fifth are in the 30-39 $(n=8 ; 19.0 \%)$ and the same proportion for those who are in the 47-54. More than a half are males ( $n$ $=22 ; 52.4 \%)$ and less than a half are females $(n=20 ; 47.6 \%)$. Less than a half are Bachelor degree nurses $(n=$ $20 ; 47.5 \%)$, more than two fifth are associate nurse $(n=18 ; 42.9 \%)$, and a small proportion are physicians $(n=$ $4 ; 9.5 \%)$. The most have Bachelor degree education $(n=24 ; 57.1 \%)$ and two fifth have Diploma degree $(n=$ $18 ; 42.9 \%$ ). The Mean years of employment is $13.7 \pm 8.5$; more than a two fifth have $\geq 16$ years of employment $(n=17 ; 40.5 \%)$, less than a quarter have $\leq 6$ years of employment $(n=10 ; 23.8 \%)$, a fifth have $7-10$ years of employment $(n=8 ; 19.0 \%)$, and less than a fifth have $11-15$ years of employment $(n=7 ; 16.7 \%)$. The Mean years of working in current unit is $5.1 \pm 3.4$; the most have $\leq 5$ years of working in current unit $(n=25 ; 59.5 \%)$, more than a third have $6-10$ years $(n=15 ; 35.7 \%)$, and a small proportion have $\geq 11$ years $(n=2 ; 4.8 \%)$.

Table 2. Mean and Standard Deviation for Health Promotion Standards

\begin{tabular}{|l|l|l|}
\hline Domain & Mean (SD) & Evaluation \\
\hline 1. Health Promotion Policy & $2.4 \pm 1.6$ & This matter was not considered \\
\hline 2. Health Promotion Structure & $2.9 \pm 1.4$ & This matter is worked on \\
\hline 3. Health Promotion Planning & $3.3 \pm 1.5$ & This matter is worked on \\
\hline 4. Health Promotion Objectives & $3.6 \pm 1.5$ & This matter is worked on \\
\hline 5. Health Promotion Implementation & $3.1 \pm 1.4$ & This matter is worked on \\
\hline 6. Health Promotion Evaluation & $3.1 \pm 1.3$ & This matter is worked on \\
\hline Overall Health Promotion Standards & $18.4 \pm 5.4$ & This matter is worked on \\
\hline
\end{tabular}

The Mean of the standard of health promotion objective has the highest value $(\mathrm{M}=3.6 \pm 1.5)$, followed by the standard of the health promotion planning $(\mathrm{M}=3.3 \pm 1.5)$, the standard of health promotion evaluation $(\mathrm{M}=3.3 \pm 1.3)$, the standard of health promotion implementation $(\mathrm{M}=3.3 \pm 1.4)$, the health promotion structure $(\mathrm{M}=2.9 \pm 1.4)$, and lastly the health promotion policy $(\mathrm{M}=2.4 \pm 1.6)$.

Table 3. Standards and the Overall Health Promotion Levels of the Hospitals $(\mathrm{N}=42)$

\begin{tabular}{|l|l|l|l|l|l|l|l|l|}
\hline Hospital Name & $\begin{array}{l}\text { H. P. } \\
\text { Policy }\end{array}$ & $\begin{array}{l}\text { H. P. } \\
\text { Structure }\end{array}$ & $\begin{array}{l}\text { H. P. } \\
\text { Planning }\end{array}$ & $\begin{array}{l}\text { H. P. Object- } \\
\text { ives }\end{array}$ & $\begin{array}{l}\text { H. P. Implement- } \\
\text { ation }\end{array}$ & $\begin{array}{l}\text { H. } \\
\text { Evaluation }\end{array}$ & $\begin{array}{l}\text { Overall } \\
\text { Evaluat- } \\
\text { ion }\end{array}$ \\
\hline $\begin{array}{l}\text { Al-Diwaniyah } \\
\text { General }\end{array}$ & 1 & 2 & 4 & 1 & 2 & 1 & 11 \\
\hline $\begin{array}{l}\text { Al-Haboobi } \\
\text { Teaching Thi-Qar }\end{array}$ & 3 & 3 & 4 & 4 & 4 & 2 & NHP \\
\hline $\begin{array}{l}\text { Al-Hillah Teaching } \\
\text { Hospital }\end{array}$ & 3 & 2 & 6 & 4 & 4 & 4 & NHP \\
\hline $\begin{array}{l}\text { Al-Hussain } \\
\text { Teaching Karbala }\end{array}$ & 2 & 2 & 5 & 2 & 4 & 2 & NHP \\
\hline
\end{tabular}


Evaluation of Health Promotion Standards in the Iraqi Teaching Hospitals: A National Survey

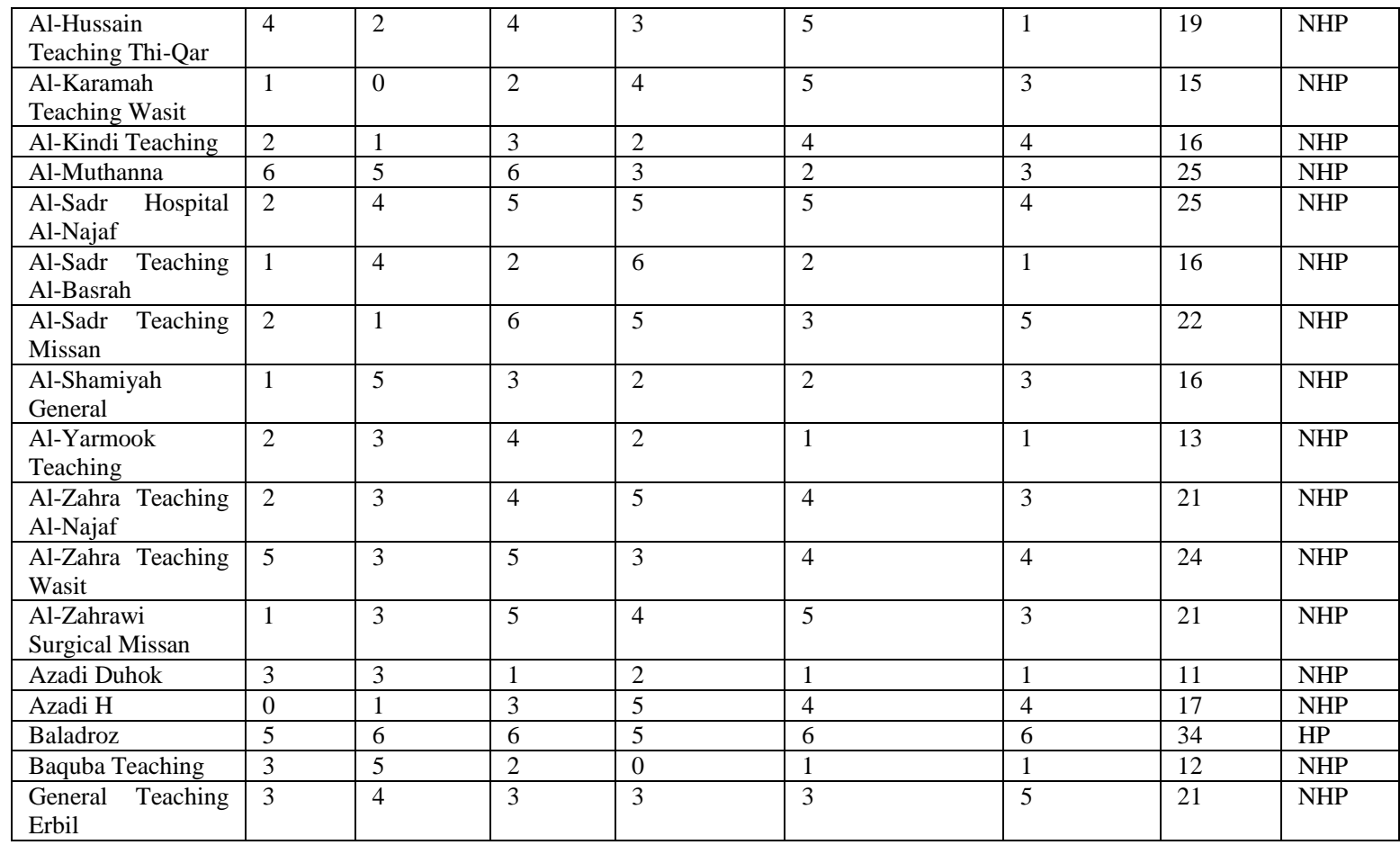

Table 3. (Continued)

\begin{tabular}{|c|c|c|c|c|c|c|c|c|}
\hline Hospital Name & H.P. Policy & H.P. Structure & H.P. Planning & H.P. Objectives & H. P. Implementa-tion & H.P. Evaluation & Overall & Evaluat-ion \\
\hline General Teaching Sulaimaniyah & 0 & 2 & 3 & 5 & 2 & 3 & 15 & NHP \\
\hline Genral Al-Basrah & 2 & 4 & 4 & 5 & 5 & 3 & 23 & NHP \\
\hline Ghazi Al-Hareen & 6 & 6 & 4 & 4 & 5 & 4 & 29 & $\mathrm{HP}$ \\
\hline Heart Disease Missan & 1 & 1 & 2 & 1 & 2 & 1 & 8 & NHP \\
\hline Hevi H Duhok & 1 & 1 & 0 & 3 & 3 & 1 & 9 & NHP \\
\hline Ibn Al-Beetar & 3 & 4 & 5 & 3 & 3 & 3 & 21 & NHP \\
\hline Ibn Al-Nafees & 4 & 3 & 1 & 3 & 5 & 5 & 21 & NHP \\
\hline Jimhoree G & 3 & 2 & 2 & 4 & 2 & 4 & 17 & NHP \\
\hline Karbala Teaching Hospital & 3 & 3 & 3 & 5 & 4 & 4 & 22 & NHP \\
\hline Kirkuk G & 3 & 2 & 2 & 5 & 2 & 4 & 18 & NHP \\
\hline Matemal Child Al-Muthanna & 5 & 3 & 3 & 3 & 0 & 3 & 17 & NHP \\
\hline Merjan Teaching Hospital & 2 & 2 & 3 & 5 & 4 & 5 & 21 & NHP \\
\hline Neuroscience Hospital & 4 & 4 & 5 & 4 & 3 & 3 & 23 & NHP \\
\hline Pediatric Baghdad & 1 & 4 & 1 & 0 & 2 & 2 & 10 & NHP \\
\hline Rezgaree Dohuk & 3 & 2 & 2 & 5 & 2 & 4 & 18 & NHP \\
\hline Rezgan Erbil & 2 & 4 & 4 & 5 & 2 & 5 & 22 & NHP \\
\hline Rozhwa Teaching Erbil & 0 & 1 & 3 & 5 & 4 & 4 & 17 & NHP \\
\hline Shar Teaching Sulaimaniyah & 1 & 3 & 1 & 4 & 3 & 4 & 16 & NHP \\
\hline Shorawo $\mathrm{H}$. & 2 & 3 & 2 & 5 & 4 & 4 & 20 & NHP \\
\hline Shorsh H. & 1 & 3 & 3 & 3 & 2 & 2 & 14 & NHP \\
\hline Teaching Baghdad & 1 & 1 & 3 & 3 & 2 & 2 & 12 & NHP \\
\hline
\end{tabular}

HP = Health Promoting; NHP = Non-Health Promoting

The vast majority of the hospitals are non-health-promoting $(n=40 ; 95.2 \%)$.

\section{Discussion}

The standards of health promotion objectives and the standards of the health promotion planning scored the highest values. On the other hand, the standards of the health promotion structure and the health promotion policy have the lowest scores. This can be attributed to that the top managers in the Iraqi health care institutions in general do not recognize the importance of putting the issue of health promotion standards into practice. Moreover, implementing the health promotion standards requires adequate and highly qualified personnel. According to the World Health Organization - EMRO, all hospitals have inadequate financial power by which they can manage the lack of some of the items by doing local procurement, but all the planning and budgeting and resource allocation is done at the central level, the hospital manager is able to plan his routine daily work but strategic planning for the hospital as to decide what services the hospital is to provide, or to close a ward or open it is a decision taken at central level. Consistently, the WHO stated that Iraq has some of the 
worst health indicators indexes in the region, practically as substandard as those of Yemen, the poorest country in the Arab world ${ }^{(12)}$.

Consistently, the Iraqi Ministry of Health $(\mathrm{MOH})$ stated that the main challenges that face the health sector in Iraq include the scanty resources, huge lack in the number of health agencies (hospitals and primary health care centers, lack of medical and allied health personnel, mass destruction of health infrastructures, absence of fair distribution of medical services among governorates, and between urban and rural areas, demographic changes among regions due to immigration which posed a critical challenge for applying the health policies, poor community participation owing to clients' dissatisfaction with quality of the delivered healthcare services, and the outdatedness of the currently followed laws and legislations which do not fit the governmental shifting toward reforming of the health system ${ }^{(13)}$. In addition to the aforementioned challenges, the poor economic status for large number of Iraqis steer them to seek the health care services they require in the governmental agencies which offer such services for nominal fees. This more probably constitute a huge burden on the governmental health agencies in terms of resources, maintenance of such agencies, and strategic planning for offering quality care through which a reliable evaluation can be implemented for the different aspects of the health care delivery system; including the health promotion standards in the hospitals.

The Iraqi Ministry of Health had formulated a new mission after 2003 which aims to improve access to quality health care irrespective of ethnicity religion or geographic origin or socioeconomic status and to improve management of the health sector ${ }^{(12)}$. Alongside with the after 2003 mission, the Iraqi MOH formulated a new vision which encompasses seven core elements of population, empowerment, community involvement, integrated health services with emphasis on primary health care, financial risk protection (equity), health provider management autonomy, quality improvement and human resource development ${ }^{(12)}$.

\section{Conclusion}

This study is the first that was conducted to shade the light on the reality of health promotion standards in the governmental teaching hospitals in Iraq. The limitations include the paucity of the relevant literature either in Iraq or the Middle Eastern region. There is a need for further research that employ another tool that uncover more thoroughly the specific health promotion standards in hospitals.

\section{Acknowledgements}

The researchers would like to express their cordial appreciations for all respondents in this study.

\section{References}

[1]. World Health Organization. The Ottawa Charter for Health Promotion 1986.2016 .72016$. <http://www.who.int/healthpromotion/conferences/previous/ottawa/en/>.

[2]. Groene, Oliver and Svend Juul Jorgensen. "Health promotion in hospitals - a strategy to improve quality in health care." The European Journal of Public Health 15.1 (2005): 6-8.

[3]. McKee, Martin and Judith Healy. Hospitals in a Changing Europe. Buckingham: Open press University, 2002. <http://www.euro.who.int/_ data/assets/pdf file/0004/98401/E74486.pdf>.

[4]. McKee, Martin. "For Debate - Does Health Care Save Lives?" Croatian Medical Journal 40.2 (1999): $123-128$. <http://www.cmj.hr/1999/40/2/10234053.htm>.

[5]. Garcia-Barbero, Milagros. "Evolution of health care systems." Feasibility, Effectiveness, Quality and Sustainability of Health Promoting Hospital Projects. Ed. Juergen M. Pelikan, Karl Krajic and Hubert Lobnig. Vienna, Austria: G. Conrad Health Promotion Publications, 1997. 27-30. 7 May 2016. <http://www.retehphitalia.it/Allegati/proceeding_5conf.pdf>.

[6]. (26) WHO. (1997) The Vienna Recommendations on Health Promoting Hospitals. WHO, Copenhagen.

[7]. World Health Organization (WHO). Iraq - Primary Health Care. July 2016.282016. <http://www.emro.who.int/irq/programmes/primary-health-care.html $>$.

[8]. World Health Organization. Healthy Settings. 2016. 2 May 2016. <http://www.who.int/healthy_settings/types/hospitals/en/>.

[9]. Maneesriwongul W, Dixon J. Instrument translation process: a methods review. Journal of Advanced Nursing [serial online]. October 15, 2004;48(2):175-186 12p. Available from: CINAHL Complete, Ipswich, MA. Accessed May 8, 2016.

[10]. Jones, Elaine G. and Margarita Kay. "Instrumentation in Cross-Cultural Research." Nursing Research 41.3 (1992): 186-188.

[11]. World Health Organization. The Ottawa Charter for Health Promotion. 2016.22016. <http://www.who.int/healthpromotion/conferences/previous/ottawa/en/>.

[12]. Jones, Patricia S., et al. "An Adaptation of Brislin's Translation Model for Cross-cultural Research." Nursing Research 50.5 (2001): 300-304.

[13]. USAID: FROM THE AMERICAN PEOPLE. Iraq: Health. 16 June 2016. 28 July 2016. <https://www.usaid.gov/iraq/health>.

[14]. The Iraqi Ministry of Health. The Electronic Strategy and Action Plan for Information Technology and Communication in Health Sector. 2011. <http://www.moh.gov.iq/upload/upfile/ar/estra.pdf>. 